$\mathrm{C}$ du trèfle frais varie de 50 à 150 , compté sur l'extrait sec; le contenu en vitamine $\mathrm{C}$ du fourrage préparé avec du trèfle, déterminé dans plusieurs dizaines d'échantillons, varie de 40 à 150. Le contenu en vitamine $\mathrm{C}$ du trèfle sec ainsi que celui du foin n'est en général que $0-2$.

Nous avons suivi le contenu en vitamine A, en déterminant la quantité de carotène. Les résultats obtenus jusqu'à présent ont prouvé que le carotène se conserve entièrement dans le fourrage A. I. V. Puisque le carotène n'est pas soluble dans l'eau, le fourrage ne subit aucune perte en vitamine A par le pressage du jus.

\title{
UN MODE SIMPLE, PRATIQUE ET RATIONNEL DE PAIEMENT DU LAIT EN FROMAGERIE.
}

par

M. E. VAILLANT

I'génieur agronome.

(Fin.)

\section{LA QUESTION DE LA PROPRETÉ ET DE LA FRAICHEUR DU LAIT.}

Afin de répondre tout de suite à une très sérieuse objection qui pourrait nous être formulée, nous croyons utile de dire que nous approuvons de toutes nos forces la suggestion de MM. Porcher et VITOUx et en général de tous ceux qui s'intéressent à l'amélioration tant désirée de la propreté du lait, tendant à faire entrer en ligne de compte dans l'évaluation du prix du lait, cette notion de propreté et aussi celle de la fraîcheur.

Nous concevons d'autant mieux l'importance de cette question que nous nous sommes ici placé au point de vue de la fromagerie, où chacun sait que la qualité des produits fabriqués dépend particulièrement de ces deux qualités du lait employé. Aussi paraît-il urgent et nécessaire de rechercher les moyens de provoquer un pas en avant dans cette voie, en faisant subir, si possible, au prix du lait des plus- ou moins-values selon que celui-ei sera reconnu propre ou malpropre.

La solution pratique de ce problème est beaucoup plus ardue à trouver que la précédente, non pas qu'on soit dépourvu de certains moyens assez commodes d'appréciation et de vérification, mais surtout parce que la fixation des moins-values à appliquer ne peut trouver de bases réelles et indiscutables.

Mais il y a une autre difficulté, d'ordre moral celle-là, qui est pour ainsi dire insurmontable dans la plupart des cas; on ne la fait 
jamais entrer en ligne de compte et cependant elle a une très grosse importance.

Nous avons dit, au cours de ce travail, que les industriels voyaient de plus en plus surgir dans leur proche voisinage soit des industriels concurrents, soit des centres de ramassage de grosses sociétés laitières, soit enfin des coopératives laitières.

Sans nous appesantir sur la rigueur plus ou moins grande que pourraient apporter ses concurrents industriels dans l'achat du lait, disons tout de suite que les coopératives sont, de par leur constitution et leur organisation, dans la quasi-impossibilité de tenir compte dans le règlement du lait à leurs adhérents de la propreté et de la fraîcheur de celui-ci. Non pas, certes, qu'elles ne devraient pas le faire, mais elles ne le peuvent... Pourquoi? Tout simplement parce que ce sont les fournisseurs de lait eux-mêmes qui dirigent l'organisme et qu'ils sont paralysés totalement par la crainte des mécontents et l'effroi des représailles possibles... Et puis, ces dirigeants fournissent-ils toujours, eux-mêmes, du lait irréprochable... !

Tout industriel qui, en conscience, par souci de bien faire et aussi d'être juste et loyal, appliquerait sans trembler le régime des moins-values au lait malpropre ou acide, serait immédiatement mis à l'index et verrait bientôt ses fournisseurs le quitter pour aller vers ceux qui sont plus conciliants que lui.

Et voilà pourquoi en France on rencontre si peu de laiteries où ces deux qualités du lait soient prises en considération et où elles fassent l'objet d'une modification du prix du lait.

On se contente en général de refuser timidement les laits franchement tournés, et e'est tout.

Nous ne voulons cependant pas nous laisser arrêter par ces arguments, conclure qu'il n'y a rien à faire et que, par conséquent, aucune méthode n'a chance d'être un jour prise en considération... S'il én était ainsi, il faudrait admettre que notre industrie veut, de gaîté de cœur, courir au suicide...

N'ayons pas crainte de dire que ce qui fait la force de certains de nos dangereux concurrents étrangers, c'est que, grâce à une diseipline de fer qui put être imposée à la production par la force d'immenses organisations englobant tout le territoire d'un Etat, et avec l'appui officiel de celui-ci, ils ont pu arriver à une qualité du lait telle que les produits fabriqués avec lui atteignent presqu'à la perfection.

Lorsqu'en France on aura mis fin à la dissémination scandaleuse des efforts, lorsque toute l'industrie laitière, sans distinction de forme, d'affinité ou de tendance, aura compris qu'une union totale, complète de tous doit se faire pour exiger partout, sans aucune 
exception, la fourniture d'un lait propre et sain sous peine de représailles convenues à l'unanimité, notre industrie pourra espérer prendre la première place en Europe, à laquelle elle a droit.

Ceci dit, voyons brièvement les données du problème et la solution qu'il est possible d'envisager.

\section{PROPRETE DU LAIT.}

\section{a) Que doit-on entendre par la propreté du lait ?}

Si l'on voulait pousser à fond la question, on pourrait tout simplement qualifier "malpropre " un lait qui contiendrait soit des matières inertes en suspension, soit des microbes ou des moisissures.

Pour la commodité de notre exposé, nous réserverons seulement la qualification "malpropres" aux laits contenant des matières inertes, La question microbienne étant plutôt en relation directe a vec la fraîcheur du lait, nous en parlerons plus loin.

Il y a deux sortes de laits " propres ».

Ceux qui le sont naturellement parce qu'ils ont été récoltés avec toutes les précautions d'hygiène désirables sur des animaux sains et propres.

Et ceux qui le sont artificiellement parce qu'ils ont été soigneusement filtrés après la traite, mais qui primitivement étaient plus ou moins malpropres.

Les premiers sont certainement des laits excellents à tous les points de vue.

Les seconds peuvent très rapidement devenir inutilisables, en raison de leur contamination certaine par les microbes apportés à l'origine par les impuretés.

\section{b) Comment déceler et mesurer les malpropretés du lait ?}

Il existe des appareils très pratiques, appelés "lacto-filtreurs " ou encore "lacto-sédimentateurs à ouate ", qui sont communément utilisés dans les laboratoires industriels.

Le plus répandu consiste en une bouteille sans fond, d'une contenance de $500 \mathrm{~cm}^{3}$, renversée, le goulot en bas, sur un support. Le goulot est fermé par une rondelle d'ouate maintenue par un dispositif métallique approprié. Cette rondelle a un diamètre de 30 à 35 millimètres.

Un échantillon d'un demi-litre de lait à analyser est versé dans la bouteille; il traverse immédiatement l'ouate pour s'écouler dans un récipient placé sous la bouteille. L'ouate joue le rôle de filtre et retient à sa surface toutes les impuretés solides. Il suffit de l'enlever après l'opération pour constater qu'elle porte un cercle plus ou moins foncé formé par toutes ces impuretés accumulées. Une échelle de teintes à laquelle on compare celle de la rondelle permet de caractériser le degré d'impuretés de ce lait. 
Des fabricants d'appareils ont établi dans ce but des tableaux de 6 ou 8 disques-types qui correspondent à la gamme allant du lait tout à fait propre au lait très sale.

Industriellement parlant, on peut reprocher à ce procédé d'exiger un temps relativement long; aussi, des constructeurs étrangers de matériel de laboratoire pour laiteries ont-ils ces derniers temps lancé des appareils basés sur le même principe que le précédent, mais permettant de faire des dosages en série et très rapidement.

On utilise dans les laboratoires scientifiques d'autres méthodes de dosage des impuretés du lait, mais à notre connaissance, il n'y en a aucune qui puisse rivaliser avec la précédente pour sa commodité et sa rapidité, et être désignée à l'attention des industriels.

Disons en terminant, que la propreté ou la malpropreté d'un lait est relativement constante, pour la simple raison qu'elle est avant tout la conséquence du tempérament et des habitudes d'hygiène $\mathrm{du}$ producteur. Un cultivateur méticuleux et soigneux aura toujours un lait propre ; un cultivateur négligent et sale par nature fournira certainement un lait malpropre, bien souvent non filtré.

Aussi, les dosages pourront-ils être espacés, afin de ne pas compliquer le service de la laiterie: un par quinzaine suffira largement, à notre avis.

\section{c) Comment peut-on faire varier le prix du lait en raison de sa propreté ?}

La malpropreté du lait étant la principale cause de sa contamination, il est évident qu'il faut, à tout prix, lutter énergiquement contre ce défaut. Cela est d'autant plus aisé que le seul responsable, en la circonstance, c'est le producteur lui-même. Le moyen le plus efficace est de l'atteindre financièrement en lui faisant subir les conséquences de son inertie et de sa négligence sous la forme d'une retenue sur sa paie de lait.

Pas de pitié pour les laits malpropres, car il est inadmissible, à l'époque où nous vivons, qu'un cultivateur soit à ce point coupable, qu'il n'ait même pas la pudeur de livrer un lait filtré.

Afin de nous tenir toujours à la simplicité et à la rapidité exigée par les industriels, surchargés de travail par ailleurs, nous eonseillons de réduire à quatre la gamme des rondelles-types devant servir à apprécier la propreté du lait :

Rondelle 1. - Immaculée. Lait parfaitement propre (ou parfaitement filtré).

Rondelle 2. - Teinte très pâle (appelée dans le jargon de la mode teinte " isabelle »). Lait assez propre. 
Rondelle 3. - Teinte gris-fauve. Lait sale, mal filtré.

Rondelle 4. - Teinte brun-noir. Lait franchement malpropre, non filtré.

Les deux premiers cas ne seront pas passibles de moins-values. Nous dirons même que l'industriel devrait si possible, et ce serait un sentiment à encourager, donner au premier une légère plusvalue, à la condition toutefois que ce lait soit frais, comme nous le verrons tout à l'heure. Ce serait un encouragement salutaire qui provoquerait l'émulation et ferait faire un grand pas dans l'amélioration de la production.

Les deux autres cas feraient l'objet de moins-values proportionnelles : faible pour le troisième cas, forte le quatrième.

Le point délicat est de chiffrer ces plus ou moins-values, ear il n'y a pas de bases pour les déterminer. A première vue, il apparaît logique que les industriels les fixent une fois pour toutes à un nombre de centimes par litre.

Comme l'application de cette méthode pourra causer une petite perturbation au début dans le monde des fournisseurs, nous croyons intelligent d'agir comme suit :

Tout d'abord, et pour faire accepter l'idée d'une sélection des laits d'après leur pureté, l'industriel pourra pendant un mois ou deux, se contenter d'afficher dans son usine les rondelles d'ouate avec le numéro ou le nom du fournisseur correspondant à chacune d'elles. Nous savons que la chose a déjà été faite et qu'il en est résulté immédiatement une amélioration... L'amour-propre de certains fournisseurs fautifs a été chatouillé...

Passé ce délai, et si l'inertie des producteurs l'y oblige, l'industriel avertira ceux-ci qu'il se voit obligé d'appliquer une moins-value de n centimes par litre aux laits sales et de 2 n centimes par litre aux laits très malpropres. Il pourra adopter le procédé en vigueur à la Société anonyme du lait condensé de Lombardie, à Milan, et qui consiste à joindre à chaque paie la rondelle d'ouate collée sur un ticket individuel afin de justifier la moins-value appliquée.

Il sera prudent, au début, de fixer les moins-values à un taux assez faible, et d'attendre que la réaction salutaire se produise. Puis, lorsque l'industriel jugera qu'aucune amélioration ne se dessine plus, et que seuls demeurent les indécrottables, les malpropres par nature, qui ne méritent aucune pitié, il augmentera carrément le taux de ses moins-values sans aucune hésitation.

\section{FRAICHEUR DU LAIT.}

\section{a) Qu'entend-on par la fraîcheur du lait?}

C'est la faculté de bonne conservation d'un lait, faculté qui est 
en fonction inverse de l'acidité, donc de la teneur microbienne de ce lait.

Un lait bien frais a une odeur agréable, une saveur douce et légèrement sucrée, une faible viscosité. Il peut être transporté sans inconvénient et parvenir à l'usine en excellent état.

Un lait altéré se distingue par une odeur aigre, une saveur légèrement acide et une viscosité plus ou moins accentuée selon son degré d'altération. Lorsque l'acidité atteint une certaine limite, le lait devient sirupeux et granuleux, puis se prend en masse : il est alors totalement impropre à tout usage.

Un lait altéré au départ de la ferme arrive rarement en bon état à l'usine; presque toujours le transport intensifie son altération et il ne peut que très rarement être encore utilisakle à l'arrivée.

Un lait propre n'est pas forcément un lait frais.

Un lait propre est un lait frais lorsque la propreté est naturelle, c'est-à-dire lorsque ce lait a été récolté avec soin et aussi aseptiquement que possible.

Un lait propre peut n'être bientôt plus un lait frais lorsque la propreté est artificielle, e'est-à-dire lorsqu'une filtration soignée a enlevé toutes les impuretés qui ont pu s'y introduire au moment de la traite.

La fraîcheur du lait est encore influencée par d'autres facteurs, qu'il nous suffit d'énumérer :

10 la propreté des ustensiles destinés à le recevoir et à le transporter ;

$2^{\circ}$ la température du milieu où il a été déposé aussitôt après la récolte.

Il est inutile de rappeler ici qu'il faut refroidir énergiquement le lait aussitôt a près la traite et le maintenir à cette basse température jusqu'à son enlèvement.

\section{b) Comment peut-on révéler le degré de fraîcheur du lait?}

$\mathrm{Si}$ un simple examen sommaire permet de caractériser, dès l'ouverture d'un bidon, un lait franchement altéré et inutilisable, il n'en est pas de même lorsqu'on veut savoir si un lait est tout à fait frais ou s'il est en voie de légère altération.

Les laboratoires scientifiques possèdent de nombreuses méthodes qui permettent de connaître d'une façon précise la fraîcheur d'un lait, soit qu'ils mesurent l'acidité de ce lait, soit qu'ils en déterminent la teneur microbienne.

Ces méthodes se groupent comme suit :

acidité : dosages ou réactions colorées ;

teneur microbienne : épreuve de la réductase, épreuve de la catalase, lacto-fermentation, numération microbienne. 
$\mathrm{Au}$ point de vue industriel, nous sommes obligé de faire un choix, et pour fixer ce choix, nous ne devons pas perdre de vue qu'il est indispensable de nous placer sur le terrain essentiellement pratique. Or, si nous nous substituons un instant à l'industriel, qui, aux premières heures de la journée, se trouve face à face avec des centaines de pots de lait qui lui arrivent par camionnées, nous devons done envisager un moyen extrêmement rapide d'appréciation qui soit simple et qui ne retarde pas le travail, car il ne faut pas oublier qu'à l'inverse du dosage de la propreté du lait, l'épreuve de la frâ̂cheur doit être faite tous les jours et sur tous les pots.

Donc, pas d'appareils compliqués, pas de longues manipulations, pas de dosages précis, mais une touche rapide suffisante pour atteindre le but proposé.

Puisque nous venons de parler de " touche " nous sommes obligé de convenir qu'il n'y a que ce procédé pour offrir la rapidité exigée.

On connaît la touche appelée aussi " goûtage " ou "dégustation ", très répandue dans notre industrie laitière, mais dont l'usage devrait être interdit parce que cette méthode est écœurante et contraire aux plus élémentaires notions d'hygiène.

M. J. Rennes, dans son livre "La question du lait " (1), l'a décrite en quelques lignes d'une saveur particulière :

“ Les hommes chargés de recevoir le lait à son arrivée trempent " leurs doigts successivement dans tous les bidons et les sucent " consciencieusement chaque fois. Cette dégustation a pour objet " de reconnaître les laits aigris et deles éliminer. Elle est donc légi" time, expéditive et peu coûteuse; mais ainsi faite, et par un procédé " si primitif, elle est dégoûtante. Elle peut même devenir dangereuse, " si les doigts ou la salive des ouvriers se trouvent souillés de germes " pathogènes, capables de se multiplier dans le lait, ce qui n'est pas " exceptionnel..." "

Pour avoir chance de succès auprès des industriels et parvenir à leur faire abandonner la méthode dont nous venons de parler, - méthode malpropre, anti-hygiénique et très imprécise, - il faut nécessairement leur en présenter une autre dont la commodité et la rapidité d'exécution soient identiques. Il est inutile de leur demander autre chose ; tous les dosages, réactions, épreuves préconisés seront réservés au laboratoire d'analyse où s'effectueront les contrôles précis, mais ils ne devront jamais en sortir.

Reste done la " touche " au papier réactif titré.

Ce papier existe-t-il ? Nous l'ignorons, mais le problème n'est pas insoluble : nous voulons en donner pour preuve les essais de M. Tapernoux relatés dans le compte rendu de la Société de

(1) J. Renves : La question du lait, Masson et Cie, éditeurs. 
Biologie (1) et portant sur l'utilisation d'un papier indicateur à base de rouge de méthyle qui de jaune à l'état normal vire au rouge orangé dans les laits acides.

Il nous semble que d'autres essais pourraient être tentés en employant, par exemple, l'alizarine, le pourpre de bromocrésol, le rouge de phénol, le bleu de bromothymol, etc. Ce n'est à notre avis qu'une question de mise au point, dont viendraient certainement à bout rapidement nos grandes maisons de produits chimiques si leur attention était sérieusement appelée sur ce sujet.

Le papier ainsi préparé serait débité en petites bandes de 5 à $6 \mathrm{~cm}$. de longueur sur $1 \mathrm{~cm} .5$ de largeur.

Le " toucheur "- qui remplacerait le "goûteur»-plongeant une bande de papier réactif dans chaque bidon pourrait, aussi rapidement qu'autrefois, opérer le triage des laits et cela d'une façon plus élégante et plus rationnelle.

\section{c) Peut-on faire varier le prix du lait selon sa fraîcheur ?}

Solutionnons tout de suite un premier cas :

Les laits franchement altérés et reconnus inutilisables parce qu'ils sont ou bien complètement tournés ou bien sur le point de l'être (laits lourds, laits visqueux et granuleux, etc.), seront éliminés d'office, refusés et retournés au producteur.

Notons, en passant, qu'une grande partie de ces retours pourraient être avantageusement évités si les ramasseurs étaient munis de papier réactif, quileur permettraient, à la ferme, et devant le fournisseur, d'écarter de suite les laits qui se révéleraient altérés. Il y aurait là une grosse économie de temps et de manipulations, laquelle n'est pas à dédaigner.

Restent maintenant les laits arrivés à l'usine et reconnus (" utilisables ». L'industriel a tout intérêt, dans le but d'éviter des fabrications défectueuses, d'opérer un triage sévère de sa matière première. Nous savons que certaines fabrications ne souffrent pas de médiocrité dans le lait, lequel doit être de première fraîcheur. Nous savons également que d'autres, comme la beurrerie, peuvent s'accommoder, dans une certaine mesure, de laits ayant subi un commencement d'altération.

S'il paraît absolument logique qu'en principe le prix du lait soit influencé par l'état de fraîcheur de ce lait, comme il a été dit relativement à sa propreté, nous sommés obligé de reconnaître qu'industriellement parlant, et dans la pratique courante des opéra tions de laiterie, il ne peut en être ainsi.

En effet, la propreté ou la malpropreté d'un lait est, comme nous l'avons vu, relativement constante. Tous les mois, au moment

(1) Le Lait, 1928, p. 836. 
de la paie, on peut avec une certitude suffisante, affirmer qu'un producteur n'a ou n'a pas suffisamment soigné ses traites et a fourni d'une façon quasi continue un produit dont tout l'ensemble était plus ou moins propre.

On ne peut affirmer, par contre, qu'une fourniture est dans l'ensemble plus ou moins altérée, et cela durant tout le mois.

On reconnaîtra bien, de temps en temps, et plutôt chez un fournisseur que chez un autre, des bidons de lait, isolés, plus ou moins altérés, mais cela ne constituera ni une généralité, ni un fait de tous les jours.

Aussi, conclurons-nous, pour le moment du moins, à l'impossibilité matérielle de faire varier le prix du lait selon sa fraîcheur. Ou bien un lait sera " utilisable » et il sera conservé, ou bien il sera "inutilisable" et il sera rendu ; aux industriels de faire montre d'une sévérité de plus en plus grande dans cet ordre d'idée : c'est la seule disposition qu'il pent prendre pour provoquer une amélioration des fournitures.

La question de fraîcheur reste, jusqu'à nouvel ordre, une question d'ordre purement technique, destinée seulementà la sélection des laits à l'intérieur de l'usine.

\section{BIBLIOGRAPHIE ANALYTIQUE}

\section{LES LIVRES}

\section{DORNER. - Methoden zur Untersuchung von Bakterien-} reinkulturen-mit einem schema zur Beschreibung von Bakterien (Méthodes de recherches des bactéries en cultures pures, avec un schéma pour la détermination des bactéries). 1933, 148 p., M. et H. Shaper, éd,, ì Hanovre. Broché 6 RM.

Le livre de DorNer constitue la traduction allemande d'une monographie intitulée : Manual of pure culture study of Bacteria, 'et publiée par la Société des Bactériologistes américains. C'est un guide précieux dans le choix des méthodes les plus estimées des savants d'outre-Atlantique.

Cet opuscule comprend huit chapitres distincts, consacrés successivement aux milieux de culture, aux procédés de coloration, à la deseription des bactéries, à l'étude des germes obligatoirement anaérobies, aux techniques expérimentales pour la mise en évidence du pouvoir pathogène des mierobes, aux méthodes sérologiques, à la détermination enfin du $p \mathbf{H}$.

Il va sans dire que le nombre de pages ne permet pas à l'auteur de décrire tous les milieux de eulture que le bactériologiste utilise au laboratoire, pour eonserver et entretenir les souches microbiennes. Le plus grand et le plus intelligent éclectisme a présidé au choix des milieux qui sont décrits : tous ont été mis à l'épreuve par une commission composée des meilleurs bactériologistes américains. 